\title{
Inter-finger Connection Matrices ${ }^{\star}$
}

\author{
Vladimir M. Zatsiorsky ${ }^{1}$, Mark L. Latash ${ }^{1}$, Fred Danion ${ }^{2}$, Fan Gao $^{1}$, \\ Zong-Ming $\mathrm{Li}^{3}$, Robert W. Gregory ${ }^{4}$, and Sheng $\mathrm{Li}^{5}$ \\ 1 Department of Kinesiology, The Pennsylvania State University, USA \\ 2 Faculté des Sciences du Sport, Université de la Mediterranée, France \\ 3 Musculoskeletal Research Center, University of Pittsburgh, USA \\ 4 School of Education, University of Kansas, USA \\ 5 Rehabilitation Institute of Chicago, USA
}

\begin{abstract}
Fingers of the hand are interdependent: when a person moves one finger or produces a force with a fingertip, other fingers of the hand also move or show force production. Hence, no direct correspondence exists between the neural commands to individual fingers and finger forces. The relations among fingers are described with inter-finger connection matrices, IFM. The IFMs depend on the number of fingers involved in the task. This presentation addresses three aspects of the IFMs: (1) computation of the IFMs, (2) role of finger interdependence during manipulation of hand-held objects and (3) inter-individual differences in the IFMs.
\end{abstract}

When a person moves one finger or produces a force with a fingertip, other fingers of the hand also move or show force production 126,4 . This phenomenon has been termed enslaving[1819]. The finger interdependence is due to three sources/mechanisms: (1) peripheral connections, both tendinous [8] and intermuscular myofascial[5], (2) multi-digit motor units in the extrinsic flexor and extensor muscles 6, and (3) central neural connections 13. Due to the enslaving, there is no direct correspondence between neural commands to individual fingers and finger forces.

The relations among fingers can be described with inter-finger connection matrices, IFM [1811]. The IFMs depend on the number of fingers involved in the task. The reason behind this dependence is a so called force deficit: a maximal force exerted by a finger in a multi-finger task is smaller than a maximal force produced by this finger in a single-finger test. The deficit increases with the number of fingers involved in the task 910]. Existence of the force deficit makes determination of the IFMs in static tasks nontrivial: recording of finger forces while the subject tries to press with only one finger does not account for the force deficit and, hence, is not sufficient to determine an IFM. This presentation addresses three aspects of the IFMs: (1) computation of the IFMs, (2) role of finger interdependence during manipulation of hand-held objects and (3) interindividual differences in the IFMs.

\footnotetext{
* This study was partly supported by NIH grants AR 048563, NS-35032 and AG-18751.
} The support from the Whittaker Foundation to Dr. Z.M. Li is also acknowledged. 


\section{Computation of the IFMs}

So far, two techniques have been used to compute the IFMs: (a) neural networking and (b) algebraic approximation.

\subsection{Neural Networks}

The three-layer network model is shown in Fig. 1. The model consists of three layers: the input layer that models a central neural drive; the hidden layer modeling finger flexors serving several fingers simultaneously, and the output layer representing finger force output. Note the existence of direct input-output connections that model muscular components that serve individual fingers. The networks incorporate the following ideas/hypotheses:

(a) Existence of two groups of muscle components. Each muscle/compartment of the first group serves an individual finger (unidigit muscles; intrinsic muscles of the hand) and each muscle/compartment of the second group serves several fingers (multi-digit muscles; extrinsic muscles of the hand). The first group of muscles is represented in the neural networks by a direct one-toone connection from the input to the output layer. The second group is represented by the middle layer and its multiple connections.

(b) The force deficit phenomenon is modeled by specific transfer characteristics of the middle layer neurons: the output of the middle layer was set as inversely proportional to the number of fingers involved. Note that in the model, the force deficit effects are only assigned to the multi-digit muscles of the hand.

(c) The enslaving effects are modeled by the connection weights from the middle to the output layer.

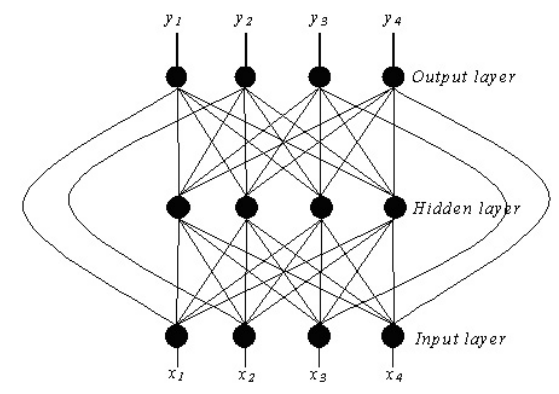

Fig. 1. Basic network. The hidden layer models the extrinsic hand muscles (those that are located in the forearm) having multiple connections to all four fingers while direct input-output connections represent the intrinsic hand muscles (those that are located in the hand) that serve individual fingers. The index, middle, ring and little finger correspond to $1,2,3$, and 4 , respectively. 
The net input to the $j^{\text {th }}$ unit of the hidden layer from the input layer is

$$
s_{j}^{(1)}=\sum_{i=1}^{4} w_{i j}^{(1)} x_{i} \quad j=1,2,3,4
$$

where $w_{i j}^{(1)}$ are connection weights from the $i^{t h}$ unit in the input layer to the $j^{t h}$ unit in the hidden layer. The characteristic of input/output in the hidden layer is described as

$$
z_{j}=f_{1}\left(s_{j}^{(1)}\right)=\frac{w_{j j}^{(1)} x_{j}}{s_{j}^{(1)}} \quad j=1,2,3,4
$$

where $z_{j}$ is the output from the hidden layer. The net input to the $k^{\text {th }}$ unit in the layer $\left(s_{k}^{(2)}\right)$ from the hidden layer is expressed as

$$
s_{k}^{(2)}=\sum_{j=1}^{4} w_{j k}^{(2)} z_{j}+v_{k} x_{k} \quad k=1,2,3,4
$$

where $w_{j k}^{(2)}$ are connection weights from the $j^{t h}$ unit in the hidden layer to the $k^{t h}$ unit in the output layer. $v_{k}$ are the connetion weights directly from the $k^{t h}$ unit in the input layer to the $k^{t h}$ unit in the output layer. An identity input/output transfer relationship was defined at the output layer, i.e.

$$
y_{k}=f_{2}\left(s_{k}^{(2)}\right)=s_{k}^{(2)} \quad k=1,2,3,4
$$

The inputs to the network were set at $x_{i}=1$, if finger $i$ was involved in the task, or $x_{i}=0$ otherwise. The weights from the input layer to the hidden layer were set as a unit constant $\left(w_{i j}^{(1)}\right)$. The network was trained using a backpropagation algorithm[1].

The developed network yielded a relation between the neural commands and the finger forces:

$$
[F]=k[w][c]+[v][c]
$$

where $[F]$ is a $(4 \times 1)$ vector of the finger forces, $[w]$ is a $(4 \times 4)$ matrix of inter-finger weight coefficients, $[c]$ is a $(4 \times 1)$ vector of dimensionless neural commands (command to a finger ranges from 1.0, when a finger is intended to produce maximal force, to 0.0 , if the finger is not intended to produce force), $[v]$ is a $(4 \times 4)$ diagonal matrix with gain coefficients that model the inputoutput relations for single-digit muscles, and $k$ is a coefficient that depends on the number of fingers in the task $(0 \leq k \leq 1)$. The value of $k$ was set either at $1 / n$, where $n$ is the number of intended fingers in the force production task, or computed by the network; the two approaches yielded similar results 18 . From (5) it follows that a command $c_{j}$ sent to a finger $j(j=1,2,3,4)$ activates all other fingers to a certain extent (enslaving effects). For a given $n$, in particular for $n=4$, (5) can be reduced to

$$
[F]=[W][c]
$$

where $[W]$ is the $(4 \times 4)$ IFM accounting for both force enslaving and force deficit $17,18,11$. 


\subsection{Algebraic Approximation}

Recently, Danion et al. 2. suggested a different equation in which the force deficit is represented by a coefficient related to the number of explicitly involved fingers and the IFM accounts only for enslaving:

$$
[F]=\frac{1}{n^{0.66}}\left[W^{\prime}\right][c]
$$

where $1 / n^{0.66}$ is an empirical force deficit coefficient and $\left[W^{\prime}\right]$ is a 'pure' enslaving matrix. The elements of $\left[W^{\prime}\right]$ can be easily estimated without the neural network computations from single-finger maximal force contraction (MVC) tasks.

The above approach inspired a mode-control hypothesis of finger coordination. According to the hypothesis, for each single-finger task, the CNS controls a unique variable (a Mode) leading to force production by the master finger, as well as by the enslaved fingers. For instance, when a subject produces force voluntarily with the index finger (I), Mode-I is recruited by the CNS. Due to the enslaving phenomenon, Mode-I also leads to force production by the middle, ring, and little fingers. Similarly, voluntary force production by the middle (M), ring $(\mathrm{R})$ or little finger $(\mathrm{L})$, is assumed to involve corresponding Modes (ModeM, Mode-R, and Mode-L, respectively). Therefore, a Mode can be viewed as a collective variable, which leads to activation of many hand muscles bringing about a specific pattern of force production by several fingers.

\section{The Role of Finger Interdependence during Manipulation of the Hand-Held Objects}

An interest to the IFMs greatly increased when it was shown that the enslaving occurs during natural grasping[17]. Knowledge of the IFMs allowed to reconstruct the intensity of neural commands sent to individual fingers and to estimate the magnitude of the enslaving effects, the force exerted by finger $i$ due to the command sent to finger $j$.

If the vector of finger forces $[F]$ and IFM matrix $[W]$ are known the vector of the neural commands can be determined by inverting (6). The vector of neural commands is then

$$
[c]=[W]^{-1}[F]
$$

To test whether the various observed force-sharing patterns were optimal, optimization methods have been employed. The norms of the following vectors were employed as cost functions:

(G1) Finger forces.

(G2) Finger forces normalized with respect to the maximal forces measured in single-finger tasks.

(G3) Finger forces normalized with respect to the maximal forces measured in a four-finger (IMRL) task. 
(G4) Finger forces normalized with respect to the maximal moments that can be generated by the fingers while grasping an object with five digits.

(G5) Neural commands.

The main distinction between the first four cost functions and the fifth one lies in the way of finger interdependence being accounted for: the cost functions based on the finger forces neglect the finger interdependence while the optimization of neural commands accounts for it.

In experiments with static holding of a handle with an attached load, when the subjects were required to produce different combinations of force and torque, some ('agonist') fingers generated moments in the direction required by the task while other ('antagonist') fingers produced moments in the opposite direction. Optimization of neural commands was able to model such 'antagonist' finger force production and resulted in a better correspondence between the actual and predicted finger forces than the optimization of various norms of the finger forces (Fig. 2, next page). Hence, during grasping strong commands to particular fingers activate also fingers that generate moments of force in the direction opposite to the direction required by the task.

\section{Inter-individual Differences in the IFMs}

Individuals forming the general population may be expected to show both similarities and differences in the way their central nervous system organizes finger interaction. These differences may be related to such gross characteristics as the amount of total maximal finger force a person can produce 15 or to more subtle individual factors such as experience with particular tasks that require precise finger coordination [16. An IFM for a hand contains 16 numbers. Hence, in general, individual differences in finger interaction, as reflected in IFMs, may require 16 variables to be fully described. We hypothesize, however, that a significantly smaller number of variables may be sufficient to describe differences among individuals without special skills. Hence, we aimed to discover such variables and relate them to indices of finger interaction introduced in earlier studies.

To this end, we recorded maximal finger forces in single-finger and multifinger tasks in 20 right-handed university male subjects. The data were analyzed in the following way:

(A) The individual IMFs were computed by the artificial neural network shown in Fig. 1. We will call these matrices the non-normalized IFMs. The sum of the elements of a non-normalized IFM equals the total force of all fingers in the four-finger task.

(B) Normalized IFMs were computed by dividing the elements of a nonnormalized IFM by the sum of its elements, i.e. by the total force of the four fingers in the IMRL task. The sum of the elements of a normalized IFM equals one. Both normalized and non-normalized IFMs were used for the further analysis. 
Supination Trial - $2.0 \mathrm{~kg}$ Load, $1.5 \mathrm{Nm}$ Torque

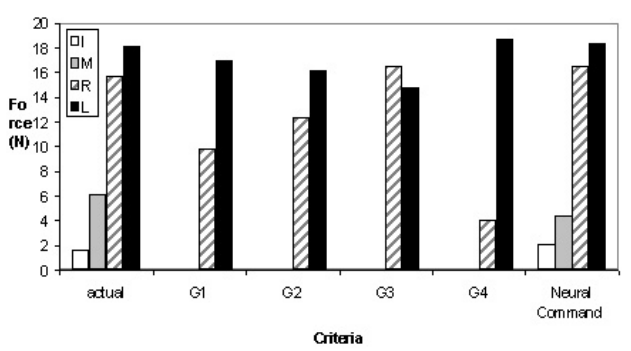

Fig. 2. Comparison of actual force data with force patterns predicted by different optimization criteria. Criteria G1-G4 do not predict the antagonist moments while the optimization of neural commands does.

(C) The similarities/dissimilarities (proximities) between the individual matrices have been quantified. Suppose we have two IFMs computed on subjects $s 1$ and $s 2$. The corresponding matrices are $\mathbf{A}$ and $\mathbf{B}$ and their difference is $(\mathbf{A}-\mathbf{B})$. The dissimilarity of the matrices was computed as a square root of the trace of a matrix, $(\mathbf{A}-\mathbf{B})^{\mathbf{T}}(\mathbf{A}-\mathbf{B})$, that is

$$
\delta_{i j}=\left\{\operatorname{trace}\left[(\mathbf{A}-\mathbf{B})^{\mathbf{T}}(\mathbf{A}-\mathbf{B})\right]\right\}^{0.5}
$$

where the superscript $T$ denotes the transpose. In order to avoid confusion with the 'distances' determined in the multi-dimensional scaling (MDS) method (see below) and following the accepted terminology, we called $\delta_{i j}$ a proximity of matrices $\mathbf{A}$ and $\mathbf{B}$, i.e., the proximity of subjects $s 1$ and $s 2$. The 190 computed values of $\delta_{i j}$ were arranged in a $20 \times 20$ proximity matrix $\Delta$.

(D) The MDS was performed on the proximity matrix. The proximity matrices $\Delta$ for both normalized and non-normalized IFM were input into MDS function module to perform MDS analyses ( Statistica, Statsoft Inc, OK, USA). The MDS program transforms the proximity data into Euclidean distances in a low-dimensional space. The Euclidean distances and the monotonic function that transforms the proximities into distances [7] were estimated by minimizing the following stress function:

$$
\text { stress }=\sqrt{\sum_{i} \sum_{j}\left[f\left(\delta_{i j}-d_{i j}\right]^{2} / \sum_{i} \sum_{j} d_{i j}^{2}\right.}
$$

where $d_{i j}$ are the distances and $f\left(\delta_{i j}\right)$ indicates a monotonic transformation of the elements $\delta_{i j}$ of the input proximity matrix $\Delta$. To estimate the goodness-of-fit the following values of stress are usually recommended: 0.2 - poor, 0.1 - fair, 0.05 - good 7 .

(E) To identify the meaning of the dimensions in the 2- and 3-dimensional spaces yielded by the MDS, individual coordinates of the subjects along the 
two/three dimensions (using both non-normalized and normalized IMFs) were correlated with the following variables: (1) sum of finger forces during four finger (IMRL) task; (2) - (5) individual finger forces during single finger (I, M, R, L) tasks, N; (6)-(9) shares of finger contribution into the total force during a four-finger task, \% (I/IMRL, M/IMRL, R/IMRL, L/IMRL); (10) location of the resultant force of the four fingers along the medio-lateral axis, mm (Location) [10]; (11) and (12) traces of the IFMs, both normalized and non-normalized, - they represent the total sum of the 'direct' finger forces, in N and percents, respectively; (13) and (14) sums of the off-diagonal elements of IFMs, both normalized and non-normalized,- they represent the total amount of the enslaved finger forces, in $\mathrm{N}$ and percents, respectively; (15) and (16) the size of the hand, its length and width, respectively.

Number of dimensions. The stress as a function of the number of dimensions (the so-called 'scree plot') is presented in Fig. 3. For non-normalized IFMs the stress can be represented sufficiently well in two dimensions (although the threedimensional representation is more accurate) while at least three dimensions are necessary to account for the individual differences in the normalized IFMs. The decrease in dimensionality achieved by the MDS was substantial: from the $20 \times 20$ proximity matrix to 2 - or 3 -dimensional spaces.

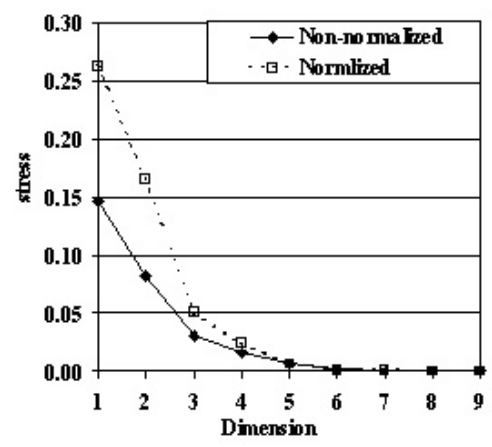

Fig. 3. Scree plots (stresses vs. number of dimensions). For non-normalized IFMs the stress was 0.098 at two dimensions and it was 0.027 at three dimensions. The stress for the normalized IFMs equaled 0.102 at two dimensions and it was 0.045 at three dimensions.

Interpretation of the dimensions. To interpret the meaning of the computed dimensions, they were regressed on a set of finger force parameters described in the text. For the non-normalized IFMs an interpretable dimension was the strength of the subjects. For the normalized IFM's, two dimensions were interpreted: (a) the location of the point of resultant force application along the medio-lateral axis that is defined by the pattern of force sharing among the fin- 
gers and (b) the total contribution of the enslaved forces into the total finger force.

In summary, the employed set of methods: experimental recording of finger $\rightarrow$ forces artificial network modeling and determining of individual IFMs $\rightarrow$ computing differences (proximities) between the individual IFMs $\rightarrow$ MDS offers promise for future research.

\section{References}

1. Bose, N.K., Liang, P.: Neural network fundamentals with graphs, algorithms and applications. McGraw-Hill, New York (1996)

2. Danion, F., Schoner, G., Latash, M.L., Li, S., Scholz, J.P., Zatsiorsky, V.M.: A mode hypothesis for finger interaction during multi-finger force production tasks. Biol Cybern 88 (2003) 91-98

3. Gao, F., Li, S., Li, Z.M., Latash, M.L., Zatsiorsky, V.M.: Matrix analyses of interaction among fingers in static force production tasks. Biol Cybern 89 (2003) 407-414

4. Hager-Ross, C.K., Schieber, M.H.: Quantifying the independence of human finger movements: comparisons of digits, hands, and movement frequencies. J Neurosci 20 (2000) 8542-8550

5. Huijing, P.: Muscular force transmission: a unified, dual or multiple system? A review and some explorative experimental results. Arch Physiol Biochem 107 (1999) 292-311

6. Kilbreath, S.L., Gandevia, S.C.: Limited independent flexion of the thumb and fingers in human subjects. J Physiol 479 (1994) 487-497

7. Kruskal, J.B., Wish, M.: Multidimensional Scaling. Sage Pub., London (1978)

8. Leijnse, J.N.: Measuring force transfers in the deep flexors of the musicians' hand :theoretical analysis, clinical examples. J Biomech 30 (1997) 873-882

9. Li, Z.M., Latash, M.L., Newell, K.M., Zatsiorsky, V.M.: Motor redundancy during maximal voluntary contraction in four-finger tasks. Exp Brain Res 122 (1998) $71-78$

10. Li, Z.M., Latash, M.L., Zatsiorsky, V.M.: Force sharing among fingers as a model of the redundancy problem. Exp Brain Res 119 (1998) 276-286

11. Li, Z.M., Zatsiorsky, V.M., Latash, M.L., Bose, N.K.: Anatomically and experimentally based neural networks modeling force coordination in static multi-finger tasks. Neurocomputing 47 (2002)259-275

12. Schieber, M.H.: Individuated finger movements of rhesus monkeys: a means of quantifying the independence of the digits. J Neurophysiol 65 (1991) 1381-1391

13. Schieber, M.H., Hibbard, L.S.: How somatotopic is the motor cortex hand area. Science 261 (1993) 489-492

14. Schieber, M.H., Poliakov, A.V.: Partial inactivation of the primary motor cortex hand area: effects on individuated finger movements. J Neurosci 18 (1998) 90389054

15. Shinohara, M., Li, S., Kang, N., Zatsiorsky, V., Latash, M.: Effects of age and gender on finger coordination in maximal contractions and submaximal force matching tasks. J Appl Physiol 94 (2003) 259-270

16. Slobounov, S., Chiang, H., Johnston, J., Ray, W.: Modulated cortical control of individual fingers in experienced musicians: an EEG study. Electroencephalographic study. Clin Neurophysiol 113 (2002) 2013-2024 
17. Zatsiorsky, V.M., Gregory, R.W., Latash, M.L.: Force and torque production in static multifinger prehension: biomechanics and control. I. Biomechanics. Biol Cybern 87 (2002) 50-57

18. Zatsiorsky, V.M., Li, Z.M., Latash, M.L.: Coordinated force production in multifinger tasks: finger interaction and neural network modeling. Biol Cybern 79 (1998) $139-150$

19. Zatsiorsky, V.M., Li, Z.M., Latash, M.L.: Enslaving effects in multi-finger force production. Exp Brain Res 131 (2000) 187-195 